Revista Med 26(2) 2018

\title{
EDITORIAL
}

\section{ENFERMEDADES HUÉRFANAS EN COLOMBIA: UNA PANORÁMICA A TRAVÉS DE LOS ERRORES INNATOS DEL METABOLISMO}

\author{
ALFREDO URIBE ARDILA M.SC, PH.D \\ ${ }^{1}$ Profesor Ciencias Básicas, Facultad de Medicina. \\ Universidad Militar Nueva Granada. \\ jesus.uribe@unimilitar.edu.co
}

Cuando se habla de enfermedades huérfanas en Colombia, generalmente las ideas giran en torno a las leyes que fueron promulgadas entre 2010 y 2011, Ley 1392 de 2010 (1) y Ley 1438 de 2011 (2), después de la fuerte presión de grupos científicos, médicos y sociedades de pacientes. Fundamentalmente se hace referencia a aquellas enfermedades crónicas y debilitantes, de naturaleza grave hasta el punto de que amenazan la vida y cuya prevalencia bordea 1 de cada 5000 personas.

Pero en la más profunda reflexión, esta definición solo fue una formalidad, para darles un estatus legal a unas enfermedades que habían sido relegadas y olvidadas dentro de los sistemas de salud en el país. Realmente lo que sucedió hace ya más de un lustro fue reconocer en el escenario de los aseguradores que, además de las afecciones comunes como país en vías de desarrollo, también teníamos dentro de nuestra casuística un problema al cual debería dársele una respuesta, que permanecía subdiagnosticado y que enfrentarlo iba a requerir un replanteamiento en las estrategias de sostenibilidad de nuestro sistema de seguridad social.

¿Pero que puedo aportar sobre estos desórdenes? Sobre la historia de las enfermedades huérfanas en Colombia y su reconocimiento en el ámbito de nuestro sistema de protección social, puedo aportar mi experiencia desde la óptica de los errores innatos del metabolismo, un grupo de enfermedades de origen genético de naturaleza monogénica, en su mayoría, cuya identificación ha crecido sustancialmente a nivel mundial en las últimas décadas y cuya incidencia acumulada abarca 1 de cada 1000 recién nacidos. Registros que han sido documentados en los países donde hay una detección sistemática a nivel neonatal, donde el diagnóstico y tratamiento precoz es parte de un programa estatal (3). Algo que en nuestro medio no existe.

Pero qué ha sucedido en Colombia con relación a los errores innatos del metabolismo. Para responder a esta pregunta se debe abordar una historia que tuvo sus comienzos a mediados de la década de 1990. Para ese entonces, en nuestro medio, estas alteraciones eran prácticamente desconocidas, lo que ofrecía un cuadro de subdiagnóstico importante, con el agravante de que muchas de las metodologías de detección estaban aún en desarrollo en centros de investigación adscritos a universidades fundamentalmente, un aspecto que no ha cambiado mucho, después de casi treinta años.

Con recursos emanados de las propias universidades y en algunos casos a través del Departamento Administrativo de Ciencia, Tecnología e Innovación (Colciencias), nacieron los primeros protocolos de detección en población de alto riesgo y se mejoraron las metodologías diagnósticas, situación que permitió lentamente estudiar enfermedades que no habían sido documentadas en la literatura nacional y que se consideraban eventos exóticos del diagnóstico médico. Esta baja frecuencia y el desentendimiento de las autoridades sanitarias dejó el concepto errado de que no deberían ser una prioridad, dentro de los programas de salud pública. 
Sin embargo, necesitarían nuestros médicos o nosotros los investigadores estar totalmente aislados, para no conocer el avance que se ha generado en los estudios sobre este tipo de alteraciones. El creciente número de publicaciones, los nuevos recursos diagnósticos, los grupos internacionales que se especializan en rutas metabólicas específicas han cambiado el panorama de estudio de estas alteraciones en Colombia, y obligado a cuestionar más la posibilidad de una alteración metabólica y a que el número de pacientes detectados muestre una frecuencia importante dentro de nuestro medio.

Son diversos y más frecuentes los reportes de casos y estudios en los que se evidencia una frecuencia importante de algunas enfermedades metabólicas en Colombia. Como experiencia personal, podría compartir los hallazgos generales del tamizaje de alto riesgo en una población de 4700 pacientes orientados clínicamente a la detección de alteraciones del metabolismo lisosomal, un recorrido de siete años de investigación (2005-2011) en que se confirmaron 242 pacientes $(5,2 \%)$ con defectos enzimáticos en el metabolismo de los esfingolípidos y glucosaminoglicanos, entre otras enfermedades (4). Una pequeña muestra de que estas alteraciones pasan desapercibidas ante los ojos de las aseguradoras y muchos pacientes mueren sin el beneficio del diagnóstico.

Quiero terminar este editorial recordando unas palabras que fueron usadas como eslogan en una de las convocatorias para el Día Mundial de las Enfermedades Raras (28 de febrero), una frase que golpea de frente y deja entrever el abandono que han sufrido estos pacientes y sus familias por los entes de protección social, porque con franqueza llevamos apenas un lustro con una ley que aún sigue en el papel, pero con más de cincuenta años de atraso en la detección sistemática de algunas de estas alteraciones, que ya son programas rutinarios en algunos países, incluso de América Latina: "Enfermedades raras... lo raro es que no nos hayan visto".

\section{Referencias}

1. Colombia, Congreso de Colombia, Ley 1392 de 2010, por medio de la cual se reconocen las enfermedades huérfanas como de especial interés y se adoptan normas tendientes a garantizar la protección social por parte del Estado colombiano a la población que padece de enfermedades huérfanas y sus cuidadores (2010 jul 2).

2. Colombia, Congreso de Colombia, Ley 1438 de 2011, por medio de la cual se reforma el Sistema General de Seguridad Social en Salud y se dictan otras disposiciones (2011 en 19).

3. Coene KL, Kluijtmans LA, van der Heeft E, et al. Next-generation metabolic screening: targeted and untargeted metabolomics for the diagnosis of inborn errors of metabolism in individual patients. J Inherit Metab Dis. 2018;41(3):337-353.

4. Uribe A, Giugliani R. Selective screening for lysosomal storage diseases with dried blood spots collected on filter paper in 4,700 high-risk colombian subjects. JIMD Reports. 2013;11:107-116. 